\title{
A cloud medication safety support system using QR code and Web services for elderly outpatients
}

\author{
Ming-Hseng Tseng ${ }^{\mathrm{a}}$ and Hui-Ching $\mathrm{Wu}^{\mathrm{b}, \mathrm{c}, *}$ \\ ${ }^{a}$ School of Medical Informatics, Chung Shan Medical University, Taichung, Taiwan \\ ${ }^{\mathrm{b}}$ School of Medical Sociology and Social Work, Chung Shan Medical University, Taichung, Taiwan \\ ${ }^{\mathrm{c} S o c i a l}$ Service Section, Chung Shan Medical University Hospital, Taichung, Taiwan
}

Received 5 December 2013

Accepted 12 January 2014

\begin{abstract}
.
BACKGROUND: Drug is an important part of disease treatment, but medication errors happen frequently and have significant clinical and financial consequences. The prevalence of prescription medication use among the ambulatory adult population increases with advancing age. Because of the global aging society, outpatients need to improve medication safety more than inpatients.

OBJECTIVE: The elderly with multiple chronic conditions face the complex task of medication management. To reduce the medication errors for the elder outpatients with chronic diseases, a cloud medication safety supporting system is designed, demonstrated and evaluated.

METHODS: The proposed system is composed of a three-tier architecture: the front-end tier, the mobile tier and the cloud tier. The mobile tier will host the personalized medication safety supporting application on Android platforms that provides some primary functions including reminders for medication, assistance with pill-dispensing, recording of medications, position of medications and notices of forgotten medications for elderly outpatients. Finally, the hybrid technology acceptance model is employed to understand the intention and satisfaction level of the potential users to use this mobile medication safety support application system.

RESULTS: The result of the system acceptance testing indicates that this developed system, implementing patient-centered services, is highly accepted by the elderly.

CONCLUSION: This proposed M-health system could assist elderly outpatients' homecare in preventing medication errors and improving their medication safety.
\end{abstract}

Keywords: Medication safety, QR code, health information technology, aged care, homecare informatics, M-health

\section{Introduction}

In developing and developed countries, aging populations are experiencing a fast growing. That is important for the elderly to maintain good medication program compliance for successful drug treatment of chronic diseases. In this interdisciplinary study of medical informatics and sociology, there is an

\footnotetext{
${ }^{*}$ Corresponding author: Hui-Ching Wu, School of Medical Sociology and Social Work, Chung Shan Medical University, No. 110, Sec. 1, J. K.N. Rd, Taichung, Taiwan. E-mail: graciewu@csmu.edu.tw.
}

0928-7329/14/\$27.50 (c) 2014 - IOS Press and the authors. All rights reserved

This article is published online with Open Access and distributed under the terms of the Creative Commons Attribution NonCommercial License. 
ambition to keep the elderly healthy and living independently, not only providing a better quality of life, but also empowering the aging population by health information technology. That is to construct a consumer-directed aged homecare, especially in medication safety promotion.

The Institute of Medicine (IOM) published a successful series of reports on quality in healthcare from 2000. The first important report To Err Is Human: Building a Safer Health System [1] reviewed the problem of medical errors requiring a focus on making systems safer rather than trying to blame individual healthcare providers. The report reminded people of the serious problem of medication safety and public awareness. As a result, substantial effort is underway to reengineer the hospital environment in a way that reduces the risk of medication errors and maximizes the quality of healthcare delivery. Crossing the Quality Chasm [2] was the second report to make four major points: errors are common and costly, systems cause errors, errors can be prevented and safety can be improved, and medicationrelated adverse events are the single leading cause of injury.

The recent report Preventing Medication Errors [3] was an attempt to think about what needs to be done to reach the next level of medication safety. The report's recommendations focused on the patient throughout the study, especially in Recommendation 3 which emphasized the need to communicate patient-specific medication information reciprocally, access safety through active monitoring, and make effective use of technologies which will vary by the setting. It provided an explicit account that patients are the center in medical health care systems. These recommendations mean that a patient can move to a different clinical setting and receive enough medication information. Combining mobile technology is the best way to achieve the goal of patient safety and assessment through active monitoring.

In the outpatient homecare environment, patients and caregivers play critical roles in protecting the safe use of medical therapies. Suitable error-prevention strategies could range from pharmacy-based and physician-based programs that help communication of medication-taking instructions to patientbased programs. The primary aim of this study was to identify the current state of medication-taking practices in community dwelling elderly who take high-risk medications, so as to identify potential targets for intervention by using information communication technologies (ICT). This study focused on the potential and preventable adverse drug events caused by errors in the medication phase. We developed a home-based M-health (mobile health) system to assist elderly outpatients to improve the medication safety and prevent medication errors.

\section{Background}

\subsection{Information technology and medication safety in aged society}

Medication errors defined as mistakes in drug ordering, transcribing, dispensing, administrating, and monitoring [4,5]. Most of the effort on medication errors has been focused on error-preventing and quality-proof systems in hospital settings [5-10]. Leape and Brennan [8] found that drug complications were the most common type of adverse event (19\%), and 58\% were errors in management. Some researches [11-15] showed that out of hospital medical errors are likely to be more common than inhospital events, but less attention has been paid to the ambulatory care setting. Errors associated with preventable adverse drug events (ADE) occurred most often at the stages of prescribing, monitoring, and errors involving patient compliance [14]. Prevention strategies should target the prescribing and monitoring stages of pharmaceutical care.

The prevalence of prescription medication use among the ambulatory adult population increases with advancing age. According to Taiwan official statistical data, the elderly (over 65 aged) constitute $11.15 \%$ 
of the population but they consumed $42 \%$ of all prescription medications in 2011 [16,17]. Every elderly person is suffering from an average of 1.4 kinds of chronic diseases. Twenty percent of the elderly use five or more medications daily. Compared with the United States in 2002, the elderly was 13\% and consumed $35 \%$ of all prescription drugs [18]. A national survey of the United States indicated that more than $40 \%$ used 5 or more different medications per week, and $12 \%$ used 10 or more different medications per week [14]. In Canada, Blais and Sears [19] reported that 20.4\% of adverse events were caused by informal caregivers. Therefore, how to improve medication safety for the elderly outpatients is an important issue.

Homecare informatics(or home telehealth) is frequently indicated the application of information technology (such as hardware, software, networks and services) for the purpose of monitoring of vital signs or to support the delivery of IT-supported homecare services [20-23]. There are important criteria for an adequate M-health technology device, including 'easily use', 'reliability', 'medication management assistance', 'routine task performance' and 'acceptability'. The elderly being initiated or maintained on medications need clear and understandable information, but current methods of communicating medication information may be inadequate [24]. Information technology can reduce the rate of errors in three ways: by preventing errors and adverse events, by facilitating a more rapid response after an adverse event has occurred, and by tracking and providing feedback about adverse events [7,25-29]. The main classes of strategies for preventing errors and adverse events include tools that can improve communication, make knowledge more readily accessible, require key pieces of information (such as the dose of a drug), assist with calculations, perform checks in real time, assist with monitoring, and provide decision support [25]. Shona et al. [30] studied the suitability of using automated Interactive Voice Response (IVR) technology to deliver a suite of cognitive assessments to the elderly using speech as the input modality. Skubic et al. [31] investigated how sensor networks for monitoring the elderly in their homes to capture patterns representing physical and cognitive health conditions and then recognize when activity patterns begin to deviate from the norm.

Computerized transcription with direct relay of entered orders to a chosen pharmacy could decrease ambulatory medication errors. Lin et al. [32] studied the feasibility of QR-code prescription in Taiwan and proved that $\mathrm{QR}$-code prescription can provide community pharmacists an efficient, accurate and inexpensive device to digitalize the prescription information. World wide web based drug information can supplement verbal information, thereby conveniently educating patients with internet access and improving drug administration [28]. In addition, personalized web pages can be created by healthcare institutions and used by patients. Kuperman et al. [33] studied the value of patient review of computerized medication records. In this research, patients added new medication data to $19 \%$ of all the 80 forms, allowing physicians to discuss discrepancies and update the computerized records. Most physicians in this research responded that the computerized medication system was useful. A trustful relationship between patients and physicians may be enhanced through information technology.

\subsection{Bar code technology in the pharmacy}

In industries, bar code technology has been widely adopted because of its ease of use and high degree of reliability. In health care system, bar code technology has been seen as a useful strategy to prevent medication errors [25,27,34]. Poon et al. studied about bar code implementation, the dispensing error rate fell $31 \%$ and the potential adverse drug events rate (the near miss rate) fell $63 \%$ in the pharmacy [35], transcription errors were completely eliminated on nursing units [36]. The technology of drug bar code indeed permitted a substantial reduction in the rate of potentially harmful errors. For the benefits of 
patient safety, in April 2006 the U.S. Food and Drug Administration (FDA) mandated bar codes for all medications used in hospitals. Many institutions are beginning to adopt this technology to increase the accuracy of the drug dispensing and administration processes.

\section{Methods}

\subsection{QR code}

In two-dimensional bar code symbols, data are encoded in both the height and width of the symbol, and the amount of data that can be contained in a single symbol is significantly greater than that stored in a one-dimensional bar code symbols [37]. The QR code (abbreviated from Quick Response Code) is a two-dimensional matrix code that belongs to a larger set of machine-readable codes. Through supported extensions, the QR code may be made up of any type of data [38]. A QR code consists of black modules (square dots) arranged in a square grid on a white background, which can be read by an imaging device (such as a camera) and processed using Reed - Solomon error correction until the image can be appropriately interpreted; data are then extracted from patterns present in both horizontal and vertical components of the image [39].

Since 1994, the QR code system has become popular outside the automotive industry due to its fast readability and greater storage capacity. Applications include manufacturing, warehousing and logistics, retailing, healthcare, life sciences, transportation and office automation. Now with the explosive growth of smartphones, the QR code is also being used in mobile marketing and advertising campaigns as a fast and effective way of connecting with customers and providing end-user content, including Web links, mobile coupons, airline boarding passes [39].

The QR codes help patients to learn about the hospital's services, get reminders about appointments, and more. Barcode squares are now moving into the clinical side of healthcare [40]. Experts predict that there will be 22 ways to use QR codes in healthcare in the future [41]. Some potential topics includes as: (1) Patient wristbands that communicate patient information quickly and easily when scanned. (2) Post-discharge follow-up: Patients that take home medical devices like ventilation machines will have QR codes on the machine that link to how-to videos on their use. (3) Temporary "tattoo" QR codes: These can be placed on dementia patients, so they can be scanned if the patient is found wandering away from home. (4) Medication lists: Doctors and hospitals can link patients' medication lists to a QR code, making it easy to access and track [40].

\subsection{Web services}

Web services were built upon XML as a train for exchanging data across applications. The basic technological infrastructure for Web services is structured around three major standards: SOAP (Simple Object Access Protocol), WSDL (Web Services Description Language), and UDDI (Universal Description, Discovery, and Integration). The standards provide the building blocks for service description, discovery, and communication. Web services technologies have clearly influenced positively the potential of the Web infrastructure by providing programmatic access to information and services. Recently, the basic Web services infrastructure was employed popularly for providing information search capabilities to an open audience (such as search engine queries, location-based services and auction monitoring.) [42]. 


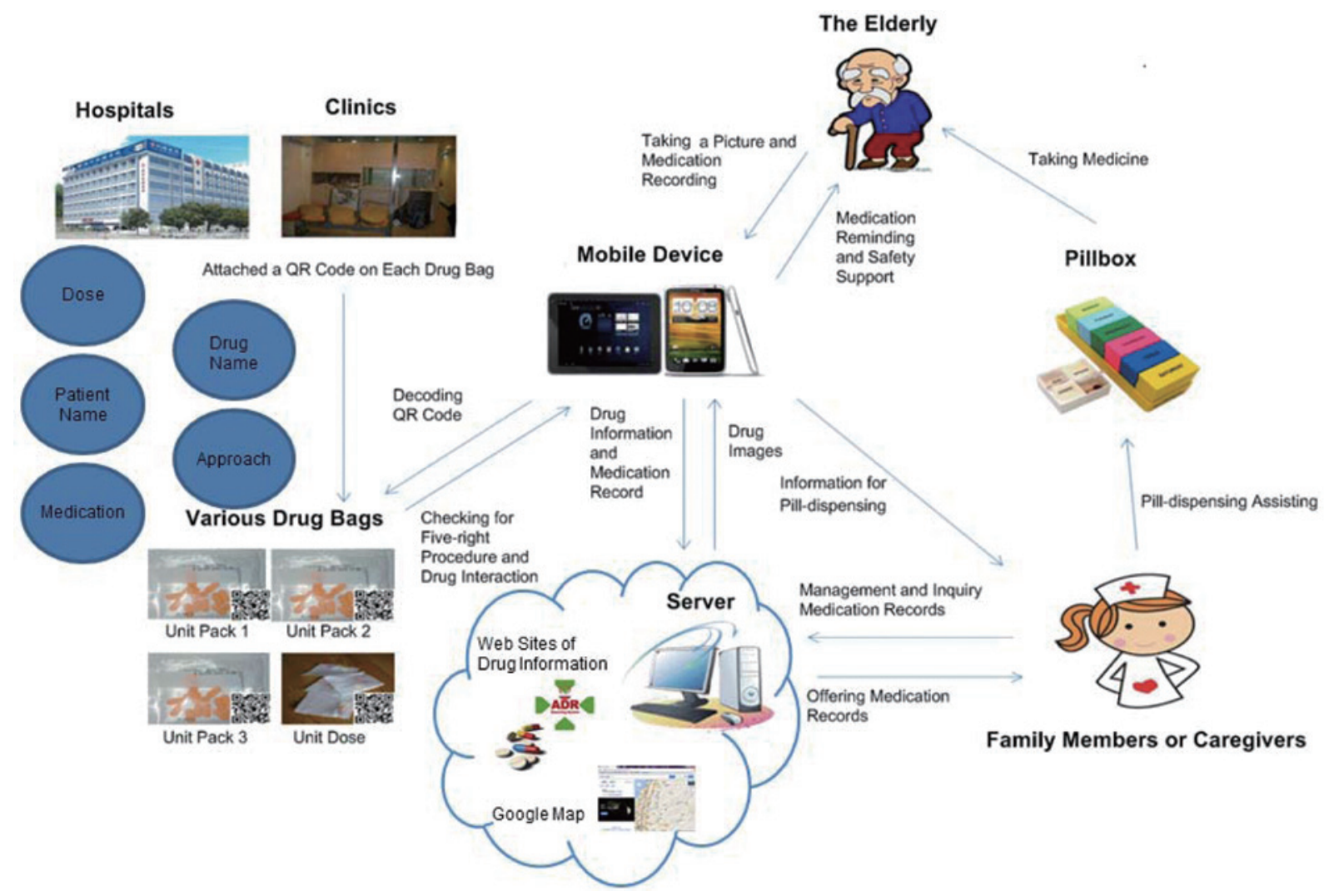

Fig. 1. System architecture. (Colours are visible in the online version of the article; http://dx.doi.org/10.3233/THC-140778)

\subsection{System design}

\subsubsection{System architecture}

The proposed system is composed of a three-tier architecture: the front-end tier, the mobile tier and the cloud tier. In the front-end tier, the hospital pharmacist or clinic pharmacist will encode a QR code with the patient's medication list attached in the drug bag by using the two-dimensional barcode generator, in which messages include: the drug-bag ID, drug-bag type, patient ID, patient name, patient sex, patient age, drug name, appearance, clinical uses, administration and dosage, and possible adverse effects. The mobile tier will host the personalized medication safety supporting application that provides some primary features including reminders for medication, assistance with pill-dispensing, recording of medications, position of medications and notices of forgotten medications for elderly outpatients with chronic diseases. Finally, there is cloud services located in the cloud tier such as: a cloud database server for storing users' medication records, Google map server for positioning the elderly, and web sites of drug information for retrieving the intended drug information by users to improve their medication safety. The proposed system architecture is illustrated in Fig. 1.

\subsubsection{Activity diagrams of mobile system and server system}

The activities involved in the mobile system are shown in Fig. 2. It focuses mainly on how to enhance the quality of personalized medication safety for the elderly with chronic diseases. First, the patient 


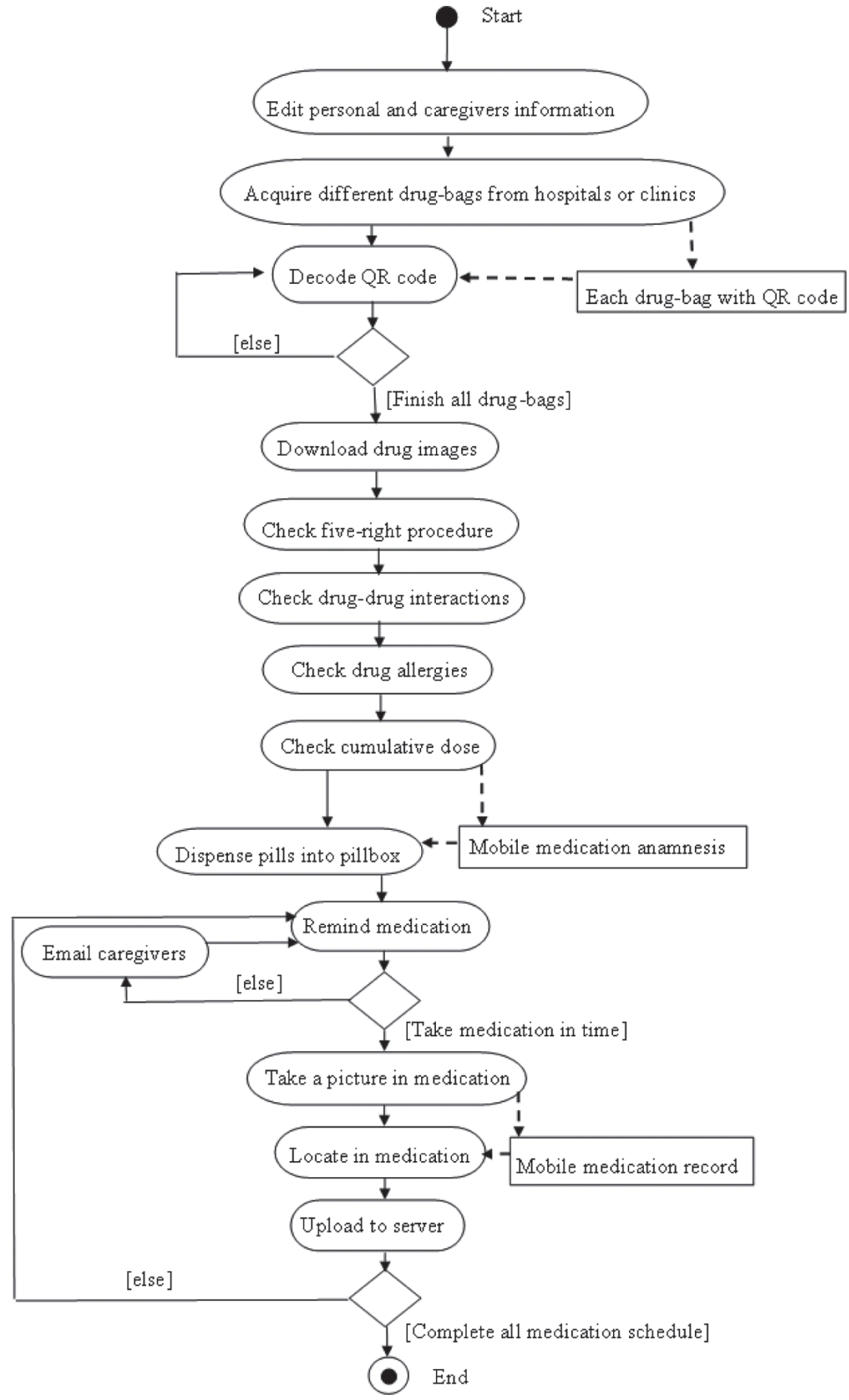

Fig. 2. Activity diagram of the mobile system. 


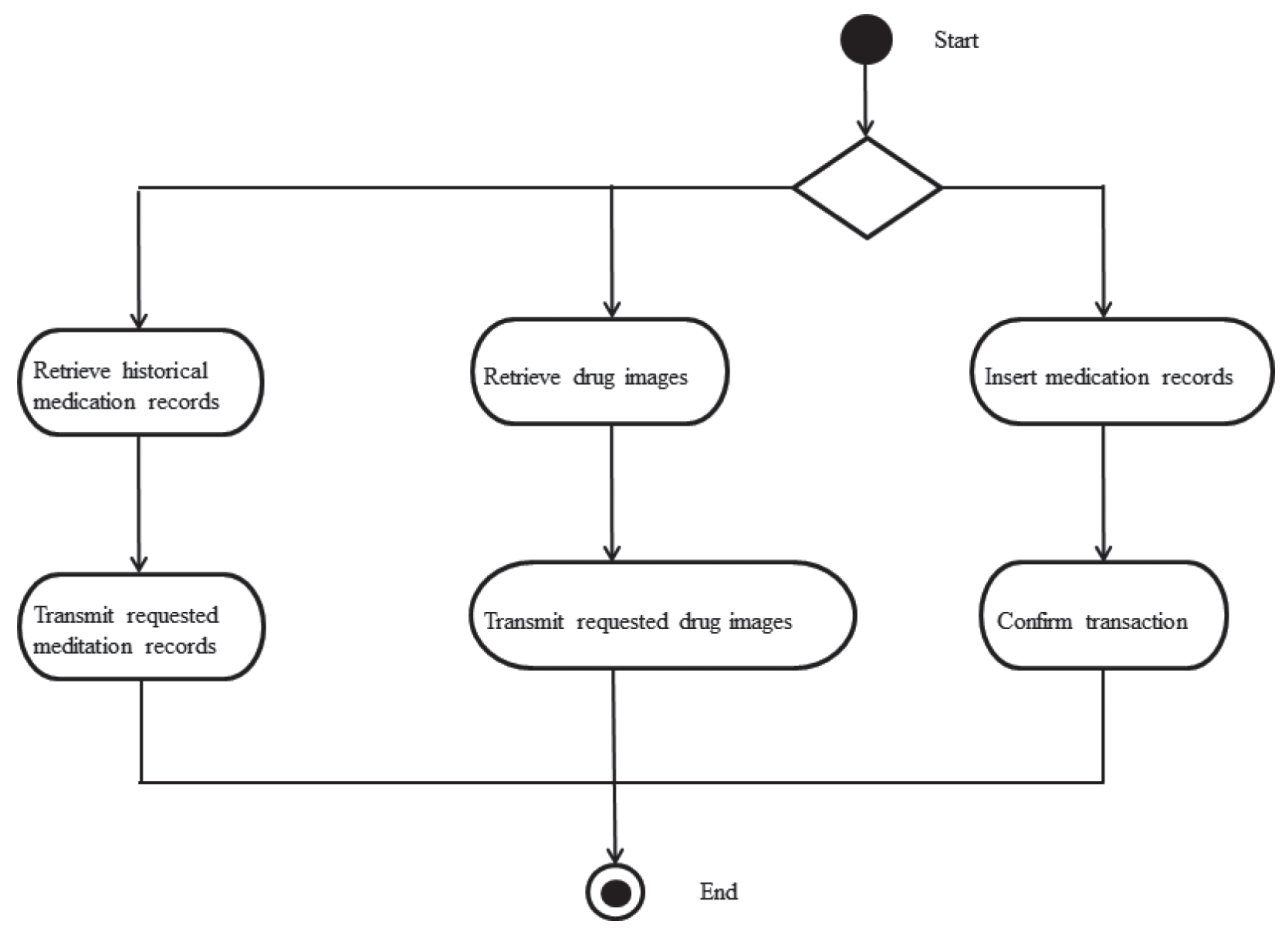

Fig. 3. Activity diagram of the server system.

has to edit her/his personal information in this application (app) system. Second, the elderly outpatients leave hospitals or clinics with a drug-bag with attached QR codes. Through the camera embedded in the smartphone, the family members or caregivers can pick up each QR code from drug bags, and then all code contents will automatically transfer into the app system. Third, through multiple medication checks provided by the mobile system, such as: drug images showing, five-rights check (right patient, drug, dose, administration route and time), drug-drug interactions check, drug allergies check and cumulative dose check, a personalized mobile medication anamnesis will be automatically generated in the app system. The multiple checks would ensure that all the medicines will be checked for potential ADEs and preventable ADEs. Medication errors could be greatly reduced, significantly enhancing the quality of the medication safety for the elderly outpatients. Fourth, the app system will assist caregivers to properly dispense pills into a small portable pillbox when the mobile medication anamnesis is done. Fifth, the app system will automatically remind the elderly patient to take medicine on time by the vibrating bell on the phone (up to four different medication times every day). It will also automatically email an announcement to caregivers about reminding the elderly to take medicine in time. Finally, the app system will generate a personalized mobile medication record with the elderly patient's picture and position after she/he took the medication, and then the medication record will be automatically uploaded to the cloud database server.

Figure 3 depicts the activities involved in the cloud server system. The database server and the web server work together as standalone hardware. The web server hosts the Web services that can be invoked by users to retrieve the intended information from the cloud database server. The server system provides three data management capabilities such that users can easily retrieve historical medication records, retrieve drug images to establish a user's drugs database in her/his smartphone, and insert new medication records for the elderly outpatients. 


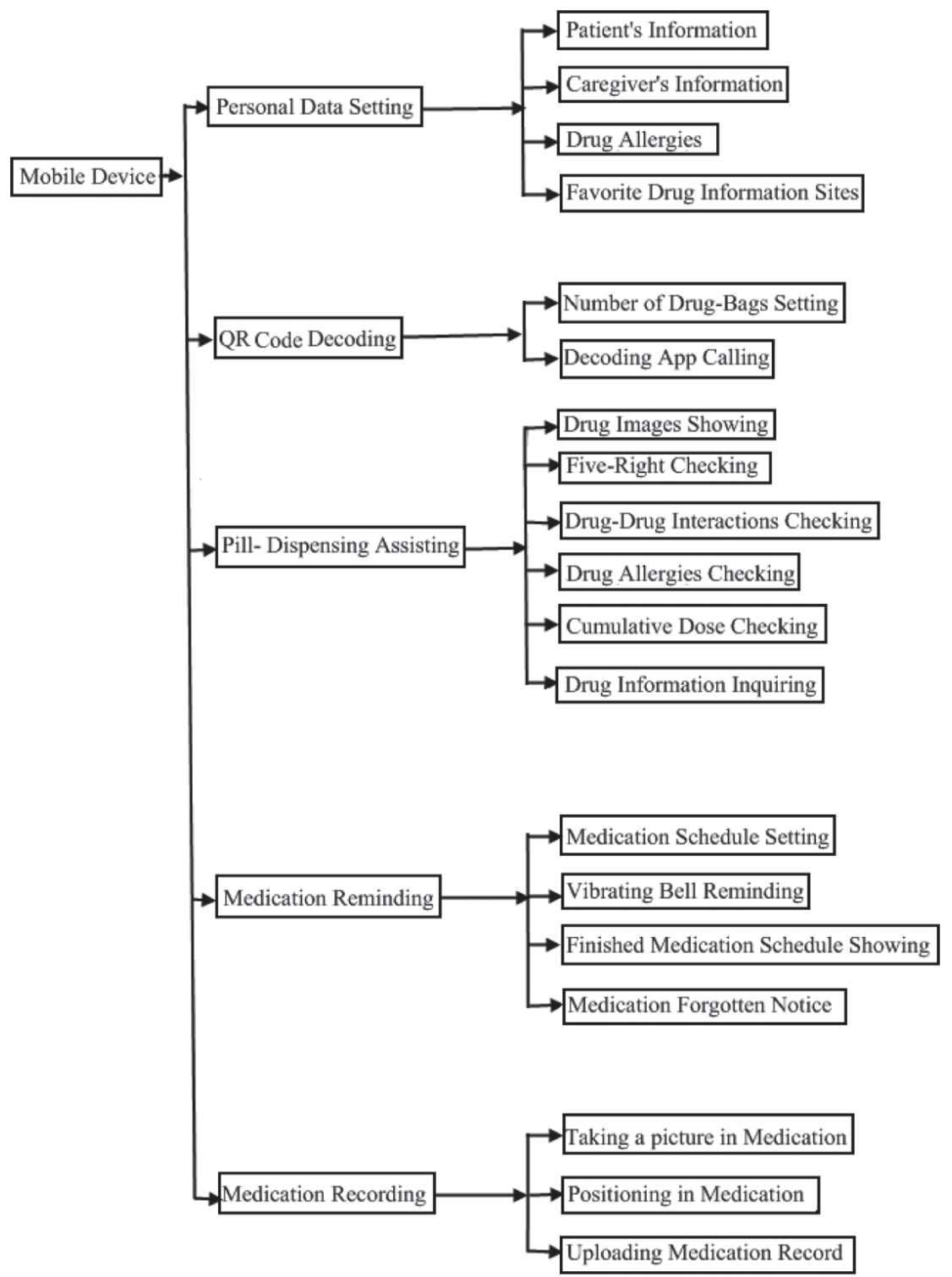

Fig. 4. Structure diagram of the mobile system.

\subsubsection{System structure of mobile system}

The structure of the mobile system is illustrated in Fig. 4. The system is structured so that it can perform five main functions. The first function is to edit the personal data setting including patient's information, caregiver's information, drug allergies and favorite drug information Web sites. The elderly outpatient, her/his family members or caregivers can link directly to these favorite drug information Web sites to obtain more medication information for enhancing the elderly outpatient's medication safety during the pill-dispensing assisting procedure.

The second function is to decode all QR codes attached on drug-bags into the existing app system. This QR-code medication information can provide an efficient, accurate and inexpensive approach to 
automatically digitalize the patient's identification and medication information and to directly transfer all data to the mobile system. The third function can assist the caregiver to properly distribute drugs to the pillbox through a series of check procedures including drug images showing, medication five rights check, drug-drug interactions check, drug allergies check, and cumulative dose check. The task of the drug appearance check is to show the positive and negative side images of every drug on the phone to help people identify medicines. The user can retrieve more web-based drug information by Internet access to supplement verbal information and improve drug administration. We think that these two functions are primary importance for enhancing outpatient's medication safety. The most important feature of the proposed system is to integrating all medication lists to generate a safe and personalized mobile medication anamnesis.

The fourth function is the reminders to take medications (a vibrating bell) according to the schedule and providing notices of forgotten medications. When the elderly outpatient forgot to take medicine on time, the app system will immediately send a medication forgotten notice E-Mail by SMTP protocol to notify the caregivers. The final function is medication recording including taking a picture and positioning when the elderly outpatient took medicine, and uploading this medication record to the cloud database server. The location of the elderly outpatient positioning is accomplished by using Google Map web service. The family members or caregivers can query the elderly outpatient's medication record from the cloud server to confirm the status of medication and her/his current position.

\subsection{System acceptance testing}

Tung et al. [43] proposed a hybrid technology acceptance model to study users' acceptance of the electronic logistics information system in the Taiwan medical industry by combining innovation diffusion theory, perceived usefulness, perceived ease of use, trust, perceived financial cost and behavioral intention to use. To understand the intention and satisfaction level of the potential users to use this medication safety support system, a preliminary evaluation of the prototype system was conducted by employing the hybrid technology acceptance model in this paper. The instrument was a seven-part questionnaire that contained basic information about the respondents, and six constructs of compatibility, perceived usefulness, perceived ease of use, trust, perceived financial cost, behavioral intention to use, and the necessity of system features. All items of the questionnaire were measured by using nominal scales to reduce the difficulty in answering for the elder patients. Table 1 lists all measurement items in this study.

\section{Results}

\subsection{Scenario demonstration}

To illustrate a scenario of the proposed system, a 68-year-old male outpatient is suffering from cardiovascular diseases and skin diseases. Figure 5 shows the QR codes attached on different drug bags obtained from a cardiology clinic and a dermatology clinic, respectively.

In order to consider the special conditions (visual degradation and the ability of mobile platform use) of the elderly, the interface of the developed mobile system is specially designed, such as simplifying all function buttons, enlarging the fonts, using font and button colors that contrast with background colors, and providing icons for every function and its progress. Figure 6 demonstrates the system interface of five main functions of the proposed mobile system. 

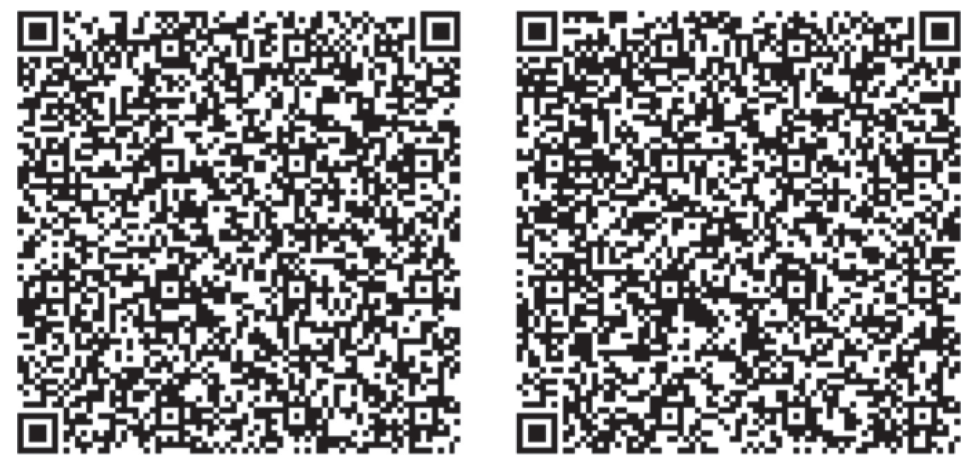

Fig. 5. Medication QR codes from two different clinics.



Fig. 6. Interface displayed for the proposed mobile system. (Colours are visible in the online version of the article; http://dx. doi.org/10.3233/THC-140778)

By following the instructions of the proposed mobile system, the elderly outpatient's family members or caregivers can help them to generate a personalized mobile medication anamnesis from the function of 'QR code Decoding' initially. Then, the caregiver can correctly distribute drugs to the pillbox for safe medication administration by using the function 'Pill-Dispensing Assisting'. Multiple medication checks are executed in this function as shown in Fig. 7. As in this scenario, the system interface displays a skull and crossbones to warn that the medication list has drug-drug interactions as depicted in Fig. 8.

\subsection{System acceptance level}

To realize the acceptance level of the users to use this medication safety support system, 20 respondents voluntarily participated in this preliminary study. Participants included 10 males and 10 females, composed of 18 patients and two caregivers. The age of patients was between 55 and 75 years old, and 40-50 years old of two caregivers. All participants were asked to use the proposed medication support system for two weeks, and then fill out the questionnaire listed in Table 1.

Table 2 shows the level of acceptability to using the medication safety support system. All of the respondents answered that they were satisfied with both constructs of perceived usefulness and trust. Ninety-five percent of participants intend to use the proposed system. The construct of perceived ease 
Table 1

Summary of measurement items

\begin{tabular}{ll}
\hline Construct & Items \\
\hline Compatibility & Using medication safety support system is suitable for my life style \\
Perceived usefulness & Using medication safety support system can improve my medication safety \\
& Using medication safety support system can enhance my drug identification \\
& Using medication safety support system can assist me in correct pill dispensing \\
& Using medication safety support system can remind me to take medicine on tome or in time \\
I find that human interface of medication safety support system is clear and easy to under- & \\
Perceived ease of use & I find that printed character of medication safety support system is clear and easy to watch \\
& I find that printed color of medication safety support system is clear and easy to differentiate \\
& I find that information display of medication safety support system is not to complex \\
Trust & As I understand it, I believe medication safety support system can help me with medication \\
& safety \\
Perceived financial cost & I think a smart phone required to deploy medication safety support system is expansive \\
Behavioral intention to use & I am willing to keep using medication safety support system \\
& I would recommend my friends to use medication safety support system \\
Necessary of system & I think the feature of automated medication information inputting is necessary \\
features & I think the feature of drug image showing is necessary \\
& I think the feature of five-right check is necessary \\
& I think the feature of drug-drug interactions check is necessary \\
& I think the feature of medication reminding is necessary \\
& I think the feature of medication record uploading is necessary \\
& I think the feature of medication photo uploading is necessary \\
& I think the feature of medication position uploading is necessary \\
I think the feature of medication forgotten notice is necessary
\end{tabular}

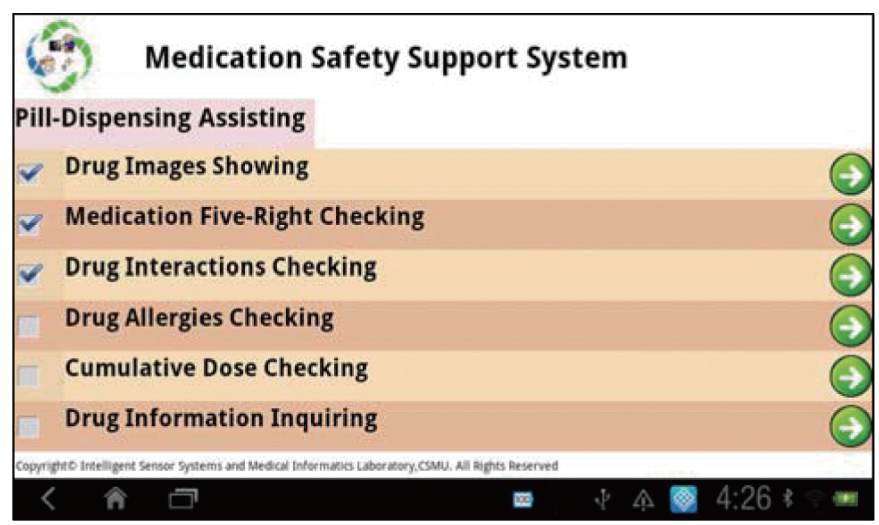

Fig. 7. Interface displayed for multiple medication checks. (Colours are visible in the online version of the article; http://dx. doi.org/10.3233/THC-140778)

of use had a $90 \%$ satisfaction level. The users thought that the printed character of the medication safety support system was not clear enough and was not easy to watch, with $85 \%$ acceptance level. A major focus in the future will be to enlarge the fonts or use large-size screen mobile devices so that the elderly patients can comfortably use the proposed mobile system. Both compatibility and perceived financial cost constructs had a lower acceptance with $80 \%$. The reason may be that five respondents did not use smartphones.

When asked about what features of the proposed mobile system are necessary, four features of 'automated medication information inputting', 'drug images showing', 'five rights checks' and 'medication 
Table 2

Acceptability to using the mobile medication safety support system

\begin{tabular}{lc}
\hline Construct & Satisfaction \\
\hline Compatibility & $80 \%$ \\
Perceived usefulness & $100 \%$ \\
Perceived ease of use & $90 \%$ \\
Trust & $100 \%$ \\
Perceived financial cost & $80 \%$ \\
Behavioral intention to use & $95 \%$ \\
\hline
\end{tabular}

Table 3

Necessity of system features

\begin{tabular}{lc}
\hline Items & Necessity \\
\hline $\begin{array}{l}\text { I think the feature of automated medication informa- } \\
\text { tion inputting is necessary }\end{array}$ & $100 \%$ \\
I think the feature of drug image showing is neces- & $100 \%$ \\
sary & $100 \%$ \\
I think the feature of five-right checks is necessary & $100 \%$ \\
I think the feature of medication reminding is neces- \\
sary \\
$\begin{array}{l}\text { I think the feature of medication forgotten notice is } \\
\text { necessary }\end{array}$ & $100 \%$ \\
$\begin{array}{l}\text { I think the feature of drug-drug interactions check is } \\
\text { necessary }\end{array}$ & $90 \%$ \\
$\begin{array}{l}\text { I think the feature of medication record uploading is } \\
\text { necessary }\end{array}$ & $85 \%$ \\
$\begin{array}{l}\text { I think the feature of medication photo uploading is } \\
\text { necessary }\end{array}$ & $80 \%$ \\
$\begin{array}{l}\text { I think the feature of medication position uploading } \\
\text { is necessary }\end{array}$ & $60 \%$ \\
\hline
\end{tabular}

\section{Medication Safety Support System}

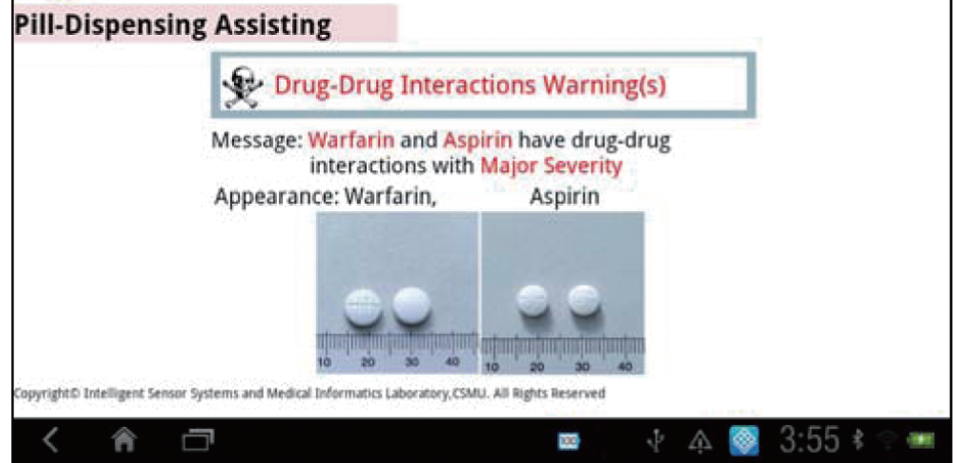

Fig. 8. Warning displayed for drug-drug interactions. (Colours are visible in the online version of the article; http://dx.doi.org/ 10.3233/THC-140778)

forgotten notice' obtained $100 \%$ level of satisfaction as shown in Table 3;90\% of participants considered 'drug-drug interactions check'; $85 \%$ of participants answered 'medication record uploading' and $80 \%$ for 'medication position uploading'; and $60 \%$ of participants think 'medication photo uploading'. One hundred percent of participates thought that the feature of 'medication forgotten notice' was necessary, but the corresponding level of acceptance in 'medication record uploading', "medication photo uploading" and "medication position uploading" was lower. Participants expressed eagerness to be concerned but also worried about their personal privacy.

\section{Conclusion}

To reduce the medication errors of the elderly outpatients with chronic diseases, a cloud medication safety supporting system on Android platforms has been developed by integrating two-dimensional 
QR code (storing medication information) and Web services (delivering drug information, medication records and outpatient's positions) techniques with a low-priced small-size hand-carry pillbox. By implementing five primary functions involving 19 tasks in the proposed application system, it can provide clearer drug information and multiple medication checks to effectively decrease the potential ADEs and preventable ADEs caused by human errors in the delivery and use of medications. Such a system would potentially improve medication safety for the elderly outpatients. The primary goal of this study is to innovate a patient-centered continued care processes and services in homecare informatics, and offer better decision support for practitioners, patients and caregivers.

We also explored the user acceptance by elderly outpatients with chronic diseases in Taiwan based on a hybrid technology acceptance model to study users' acceptance of the proposed medication safety support system. The study results supported that performance expectation has a significant and positive impact on the willingness to use the proposed mobile system. The study showed that 'perceived usefulness', 'perceived ease of use', 'trust', and 'behavioral intention to use' all had high satisfaction rates. However, 'compatibility' and 'perceived financial cost' had lower acceptance rates.

The most challenging aspects in mobile cloud healthcare system are guaranteeing user privacy and the provision of mobile application security that uses cloud resources. To protect the privacy of the users and provide a secure system environment, we will continue to improve several issues pertaining to data security, network security, data locality, data integrity, web application security, data segregation, data access, authentication, authorization and data confidentiality [44], to enhance security in the future.

\section{Acknowledgments}

This paper is partly sponsored by the National Science Council, Taiwan, ROC, under grant NSC 102-2218-E-040-003. The authors would like to thank Mr. Yu-Feng Shen, Mr. Jia-You Kang and Mr. Xing-Xian Song for helping to implement the prototype of the system.

\section{Conflict of interest}

The authors have no conflicts of interest to declare. This paper is not an advertisement for Android platforms.

\section{References}

[1] Kohn LT, Corrigan,Janet M.,Donaldson,Molla S. To Err Is Human: Building a Safer Healthcare System. In: Medicine Io, editor. Washington: National Academy Press; 2000.

[2] America IoMCoQoHCi. Crossing the quality chasm: A new health system for the 21st century. Washington: National Academies Press; 2001.

[3] Aspden P, Wolcott J, Bootman JL, Cronenwett LR. Preventing medication errors: Auality chasm series: National Academies Press; 2006.

[4] Bates DW, Cullen DJ, Laird N, Petersen LA, Small SD, Servi D, et al. Incidence of adverse drug events and potential adverse drug events. JAMA: the journal of the American Medical Association 1995;274:29-34.

[5] Leape LL, Bates DW, Cullen DJ, Cooper J, Demonaco HJ, Gallivan T, et al. Systems analysis of adverse drug events. JAMA: The journal of the American Medical Association 1995;274:35.

[6] Colt HG, Shapiro AP. Drug-induced illness as a cause for admission to a community hospital. Journal of the American Geriatrics Society 1989;37:323. 
[7] Kaushal R, Bates D. Information technology and medication safety: What is the benefit? Quality and Safety in Health Care 2002;11:261-5.

[8] Leape LL, Brennan TA, Laird N, Lawthers AG, Localio AR, Barnes BA, et al. The nature of adverse events in hospitalized patients: Results of the Harvard Medical Practice Study II. New England Journal of Medicine 1991;324:377-84.

[9] Leape LL, Berwick DM. Five Years After To Err Is Human: What Have We Learned? JAMA 2005;293:2384-90.

[10] Leape LL. Errors in medicine. Clinica Chimica Acta 2009;404:2-5.

[11] Hanlon JT, Schmader KE, Koronkowski MJ, Weinberger M, Landsman PB, Samsa GP, et al. Adverse drug events in high risk older outpatients. Journal of the American Geriatrics Society 1997;45:945.

[12] Thomas EJ, Brennan TA. Incidence and types of preventable adverse events in elderly patients: Population based review of medical records. Bmj 2000;320:741-4.

[13] Rothschild JM, Bates DW, Leape LL. Preventable medical injuries in older patients. Archives of Internal Medicine 2000;160:2717.

[14] Gurwitz JH, Field TS, Harrold LR, Rothschild J, Debellis K, Seger AC, et al. Incidence and preventability of adverse drug events among older persons in the ambulatory setting. JAMA: The journal of the American Medical Association 2003;289:1107-16.

[15] Gandhi TK, Weingart SN, Seger AC, Borus J, Burdick E, Poon EG, et al. Outpatient prescribing errors and the impact of computerized prescribing. Journal of General Internal Medicine 2005;20:837-41.

[16] Department of Household Resistration MOI. 2013.

[17] National Health Insurrance Administration MoHaW. 2013.

[18] Raehl CL, Bond C, Woods T, Patry RA, Sleeper RB. Individualized drug use assessment in the elderly. Pharmacotherapy: The Journal of Human Pharmacology and Drug Therapy 2002;22:1239-48.

[19] Blais R, Sears NA, Doran D, Baker GR, Macdonald M, Mitchell L, et al. Assessing adverse events among home care clients in three Canadian provinces using chart review. BMJ Quality \& Safety 2013;22:989-97.

[20] Miles I. Home informatics: Information technology and the transformation of everyday life. London: Pinter Publishers; 1988.

[21] Roback K, Herzog A. Home informatics in healthcare: Assessment guidelines to keep up quality of care and avoid adverse effects. Technology and Health Care 2003;11:195-206.

[22] Darkins A, Ryan P, Kobb R, Foster L, Edmonson E, Wakefield B, et al. Care coordination/home telehealth: The systematic implementation of health informatics, home telehealth, and disease management to support the care of veteran patients with chronic conditions. Telemedicine and e-Health 2008;14:1118-26.

[23] Wu HC, Tsai MC, Lin CC, Chang CJ, Chang CC, Tseng MH. Developing screening services for colorectal cancer on Android smartphones. Telemedicine and e-Health 2013; (to appear).

[24] Metlay JP, Cohen A, Polsky D, Kimmel SE, Koppel R, Hennessy S. Medication Safety in Older Adults: HomeBased Practice Patterns. Journal of the American Geriatrics Society 2005;53:976-82.

[25] Bates DW, Gawande AA. Improving safety with information technology. New England journal of medicine 2003;348:2526-34.

[26] Bates DW, Leape LL, Cullen DJ, Laird N, Petersen LA, Teich JM, et al. Effect of computerized physician order entry and a team intervention on prevention of serious medication errors. JAMA: The journal of the American Medical Association 1998;280:1311-6.

[27] Bates DW. Using information technology to reduce rates of medication errors in hospitals. BMJ: British Medical Journal 2000;320:788.

[28] Gawande A, Bates D. The use of information technology in improving medical performance. Part III. Patient-support tools. MedGenMed: Medscape general medicine 2000;2:E12-E.

[29] Huckvale C, Car J, Akiyama M, Jaafar S, Khoja T, Bin Khalid A, et al. Information technology for patient safety. Quality and Safety in Health Care 2010;19:i25-i33.

[30] D'Arcy S, Rapcan V, Gali A, Burke N, O'Connell GC, Robertson IH, et al. A study into the automation of cognitive assessment tasks for delivery via the telephone: Lessons for developing remote monitoring applications for the elderly. Technology and Health Care 2013;21:387-96.

[31] Skubic M, Gregory A, Mihail P, Marilyn R, James K. A smart home application to eldercare: Current status and lessons learned. Technology and Health Care 2009;17:183-201.

[32] Lin CH, Tsai FY, Tsai WL, Wen HW, Hu ML. The feasibility of QR code prescription in Taiwan. Journal of Clinical Pharmacy and Therapeutics 2012;37:643-6.

[33] Kuperman GJ, Sussman A, Schneider LI, Fiskio JM, Bates DW. Towards improving the accuracy of the clinical database: Allowing outpatients to review their computerized data. Proceedings of the AMIA Symposium: American Medical Informatics Association; 1998. p. 220.

[34] Bates D, Cohen M, Leape L, Overhage JM, Shabot MM, Sheridan T. Reducing the frequency of errors in medicine using information technology. Journal of the American Medical Informatics Association 2001;8:299-308.

[35] Poon EG, Cina JL, Churchill W, Patel N, Featherstone E, Rothschild JM, et al. Medication dispensing errors and potential 
adverse drug events before and after implementing bar code technology in the pharmacy. Annals of Internal Medicine 2006; 145:426-34.

[36] Poon EG, Keohane CA, Yoon CS, Ditmore M, Bane A, Levtzion-Korach O, et al. Effect of bar-code technology on the safety of medication administration. New England Journal of Medicine 2010;362:1698-707.

[37] Two-Dimensional Bar Code Overview 2013.

[38] Code.com Q. What is a QR Code? 2013.

[39] QR Code?Essentials. 2013.

[40] Jackson S. Are QR codes in your hospital's clinical future? 2012.

[41] Whaley MP. 22 Ways You Will Use QR (Quick Response) Codes in Healthcare in the Future. 2010.

[42] Benatallah B, Hacid M-S, Leger A, Rey C, Toumani F. On automating Web services discovery. The VLDB Journal 2005; 14:84-96.

[43] Tung F-C, Chang S-C, Chou C-M. An extension of trust and TAM model with IDT in the adoption of the electronic logistics information system in HIS in the medical industry. International journal of medical informatics 2008;77:32435 .

[44] Khan AN, Mat Kiah M, Khan SU, Madani SA. Towards secure mobile cloud computing: A survey. Future Generation Computer Systems 2013;29:1278-99. 